\begin{abstract}
Krasimira Hristova ${ }^{\star}$
University National Heart Hospital, Department of Noninvasive Imaging and Diagnostic, Sofia 1309, Bulgaria

Dates: Received: 07 November, 2015; Accepted: 01 February, 2016; Published: 03 February, 2016

${ }^{*}$ Corresponding author: Krasimira Hristova, MD, $\mathrm{PhD}$, Associated Professor of Cardiology, University National Heart Hospital, Department of Noninvasive Imaging and Diagnostic, Sofia 1309, Bulgaria, E-mail: khristovabg@yahoo.com

www.peertechz.com
\end{abstract}

Keywords: Atherosclerosis; Dyslipidemia; High risk patients; Noninvasive imaging

\section{Research Article \\ The Role of Noninvasive Imaging for Detection High Risk Patients with Subclinical Atherosclerosis}

\section{Abbreviations}

AI: Augmentation Index; AS: Arterial Stiffness; BP: Blood Pressure; CVD: Cardiovascular Diseases; eNOS: Endothelial NO Synthase; EPIV: Echo Particle Image Velocimetry; FMD: Flow-Mediated Vasodilatation; HDL: Chigh-Density Lipoprotein Cholesterol; IMT: Intima Media Thickness; LDL: Clow-Density Lipoprotein Cholesterol; PWV: Pulse Wave Velocity; TG: Triglycerides; us- CRP: ultra-sensitive C-Reactive Protein;

\section{Introduction}

Assessment of CVD risk relates to the availability of effective treatments that inhibit the development and progression of atherosclerosis early in the life [1]. Effective therapeutic intervention has advanced the concept of primary prevention of CAD. Primary prevention focuses on identifying asymptomatic individuals without prior history of CVD, who are at sufficiently high risk for a future CVD event to justify gradually more intensive risk reduction efforts. The causes of atherosclerosis, which are reliable for cardiovascular diseases, are among the leading of occasion for morbidity and mortality in the world. The coronary heart disease due to atherosclerosis is increasing in last years in developing countries. Chronic inflammatory diseases are associated with increasing of cardiovascular disease prevalence and outcomes of subclinical atherosclerosis and might detect the once of endothelial dysfunction by FMV method [2-4]. This phenomenon, called pathological remodeling, has been accelerated and may attribute to traditional risk factors, associated with atherosclerosis, but could also be the result of other autoimmune and inflammatory mechanisms that are aggravated in chronic inflammatory diseases and benefit in certain diseases, studied recently, such as chronic inflammatory arthritis and conjunctivitis [5].

Knowing the high prevalence of CVD and its risk factors, a major challenge is associated with primary prevention of CVD involves identification of new early risk factors as well as early diagnosis of subclinical CVD in asymptomatic individuals who are at The sudden death is the primary consequence of coronary artery disease in $50 \%$ of men and $64 \%$ of women. Historically an early detection of atherosclerosis has become eligible due to the noninvasive imaging techniques for patients with risk factors, allowing assessing and confirming plaque presence and estimating the extent of the flow limiting stenosis. This remodeling of the arterial wall is accelerated in diseases mentioned as inflammatory-related abnormalities and autoimmune mechanisms may work synergistically with traditional risk factors, making them particularly harmful and therefore contribute to endothelial dysfunction, which is considered the first step in atherogenesis.

The arterial wall is consist of 3 layers (ie, the intima, including the endothelium, the media, and the adventitia) and has individual importance in systemic circulation. The vascular endothelium settled in proper way the vascular tone, hemostasis and/or vascular permeability. The media is the major determinant of arterial elasticity, which regulates the conduit function (delivery of blood to tissues) and cushioning effect (for generation of continuous blood flow). Failure of these functions results in organ/vascular damage. The diagnosis of early changes of arterial status is possible since the development of new imaging techniques, which are commonly proposed to study not only mechanical properties but elastic properties. Except, these noninvasive methods are encouraging for assessing subclinical atheroma and the degree of it penetration by measuring the IMT, AS and the EPIV used in the calculation of the constraint of the carotid wall and of the level of endothelial dysfunction by the FMV method [6].

The screening protocol contain in a few specific biomarkers directly related to the progression of atherosclerosis (the study of metalloproteases or bio protease obtained from a sample of urine, or 
of plasma to be systematically associated with previous methods [7], let to improve the results by the end of treatment and directed by our future prognostic decisions [8]. Some of these biomarkers are still in the research stage. Still biomarkers which are using in daily practice for assessment of cardiovascular risk are LDL-C, HDL-C, TG, usCRP, glycated hemoglobin, creatinine clearance, von Will brand factor, myeloperoxidases, cytokines, and other markers of platelet reactivity. Currently the oxidative stress and calcium score (calculated by coronary computed tomography) used as a noninvasive end point in epidemiological studies and clinical trials to gauge progression and regression of atherosclerosis [9].

Accordingly, assessment of properties correlated with plaque composition is needed for detection of subclinical atherosclerosis and might become a screening tool for use in daily practice in asymptomatic patients with risk factors. In $21^{\text {st }}$ century the risk stratification is basically preventive measures aimed at reducing the occurrence of cardiovascular events [10]. Consequently, the aim of the present research is to promote the screening and early detection of subclinical atherosclerosis in asymptomatic patients with cardiovascular risk factors via mass screening or individual screening [11].

\section{Cardiovascular risk factors}

Cardiovascular risk factors (Figure 1) are modifiable parameters closely related with the outcomes of a cardiovascular occurrence that increase the probability of this event: hypertension increases the probability of death by $13 \%[12,13]$, tobacco by $9 \%$, [1416], dyslipidemia by $8 \%$ with its atherogenic effect, and by $7 \%$ malnutrition, as well as increasing the incidence of diabetes, obesity, and high body mass index, except where age remains the only nonmodifiable risk factor $[17,18]$. Some risk factors such as hypertension [19], smoking, and diabetes were assessed in multiple models for stratification of the cardiovascular risk in the general population. This hypothesis of an overall estimated risk to predict the cardiovascular risk, which plays a key role in individualized efficacy of the treatment $[20,21]$.
The earliest sings of atherosclerosis initiate among children and young people and are silent and progress slowly and first clinical display generally appear in middle age [22], although the first event can be fatal for the patient. Last years there has been an increase in recognition of the importance of subclinical atherosclerosis, especially for elderly people with subclinical disease because they have a worse prognosis than those without this disease early disease [23]. Furthermore, progression of atherosclerosis can be significantly reduced when dyslipidemia is treated by statins; reduction in cardiovascular events after statin treatment has also been widely documented [24]. Therefore, non-invasive documentation of atherosclerotic lesions has become an important objective for early treatment as well as preventive measures.

\section{Non-invasive methods of assessing vascular functions}

Endothelial function; Last 10 years the noninvasive imaging has been used in the diagnosis of subclinical atherosclerosis providing analysis of the IMT, the PWV, and the degree of endothelial dysfunction at the level of the brachial artery by the FMV method. The assessment of endothelium-dependent vasodilatation is widely used to evaluate endothelial function [25,26]. Measurements of the brachial artery diameter before and after an increase in shear stress induced by reactive hyperemia (ie, FMD) is most frequently used in the clinical setting [27,28]. During the vasodilation caused by increased shear stress in walls and by reactive hyperemia, which activates release on eNOS and increases NO production in the endothelium. FMD is a non-invasive method, which provide most robust information about a local NO bioavailability in the endothelium and play a major role factor in process of atherosclerosis [29]. Assessing FMD is possible in the daily practice [26,30]. Noninvasive assessment of the changes in arterial wall by venous occlusion plethysmography is one of the methods of vascular reactivity and releasing the prostaglandins and endothelial-derived hyperpolarizing factor, in addition to NO [30], have been helped to improve the arm blood flow [22,30]. The impaired NO production is main leading to the initiation/progression of atherosclerosis via above-mentioned

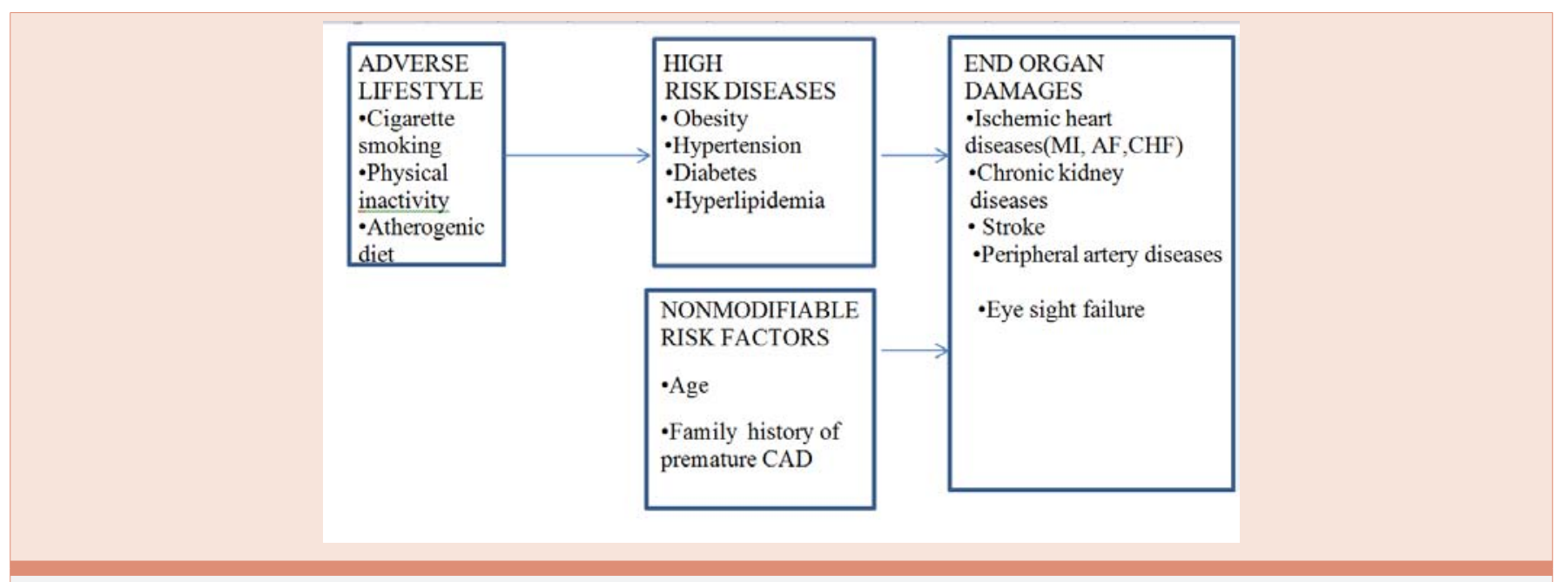

Figure 1: Major risk factors for cardiovascular diseases. 
anti-atherogenic actions of NO, like as its inhibitory effect on vascular smooth muscle cell growth, nuclear transcription of cell adhesion molecules, platelet aggregation, and leukocyte adhesion to endothelial cells [15]. Endothelial dysfunction is starting point of the vascular inflammation and that increases the production of cytokines and/ or vasoactive substances (ie, angiotensin II and endothelin-1) in the endothelium. Related complimentary to impair NO bioavailability, which causes inflammation in the vascular wall and to stiffen the arteries as functionally and structurally.

Last years were reported that FMD is a predictor of the clinical outcome in patients with CVD [30], which proposes that FMD causes plaque destabilization via local inflammation and prothrombosis activation [31]. But some of the studies are relatively small and needed a larger numbers of subjects. Some study shown that FMD provided incremental prognostic information in 2,264 post-menopausal women [32]. But in other studies this parameter as a prognostic indicator was relatively limited in 2,792 elderly subjects [33] and 842 multi-ethnic cohorts [34]. There by further studies of a larger number of subjects, especially low-risk subjects [35], are needed to verify the usefulness of FMD as a predictor of CVD outcomes.

\section{Assessment of arterial elasticity}

Pulse wave velocity (PWV): PWV represent segmental arterial elasticity in arterial wall. Contraction of the left ventricle generates a pulse wave that is propagated throughout the arterial tree [25]. PWV is measured as the distance traveled by the pulse wave divided by the time taken to travel the distance [36,37]. Increased arterial stiffness is associated with an increased propagation speed of the pulse wave in the artery. PWV can be analyzing in any arterial segment [38]. Assessment of central arterial stiffness is more relevant to CVD risk stratification than peripheral arterial stiffness. Carotid-femoral PWV (Figures 2,3) is the gold standard for assessing central arterial stiffness. Some restraint of analyzing the brachial-ankle PWV is that this marker reflects on elastic arterial stiffness and muscular arterial stiffness [39]. Nonetheless, the brachial-ankle PWV has a close correlation with aortic PWV and carotid-femoral PWV.

PWV analysis represented the degree of aging of the arterial system and its impact on the central pressure and pulse (clinical markers). The normal ranges of different parameters used by this method are for the PWV-VOP $\leq 12 \mathrm{~m} / \mathrm{s}$ (age limit 70 years) and the size of the coefficient stiffness parameters $(\alpha, \beta): \alpha \geq 11$ and $\beta \geq 20$. The measurement technique is first performed by ultrasound on common carotid artery with a $7.5 \mathrm{MHz}$ transducer in two-dimensional (2D) mode [40]. The measuring window is placed with the line $1 \mathrm{~cm}$ from the bulb, with three alternating $15-\mathrm{mm}$ measurements taken on each common carotid $[22,41,42]$. The Color Doppler should be parallel to the artery walls, remaining synchronous to the kinetics of analyzing wall.

The curve of the PWV must be fluently without a hitch and keep regular for analyzing for 10-12 seconds. The process stops at the moment the standard deviation is $<5[43,44]$ (Figure 4).

The carotid-femoral PWV were analyzed in healthy subjects, hypertensive subjects too and has been shown to be a forecast of future cardiovascular events $[45,46]$. In studies with patients with acute coronary syndrome and chronic renal disease [47] or heart failure [48], the brachial-ankle PWV has been shown as a predictor of the prognosis for cardiovascular events [49]. Aging, BP and other atherogenic factors reflected in the vascular damage that cause different level of structural stiffening of the arterial wall [50]. Increasing of the BP develop an arterial wall gradient, reasoning in functional stiffening of the arteries [51]. Commonly, PWV is widely used as predictor of atherosclerotic vascular damage, but increased $\mathrm{BP}$ is a disturbing variable, causes extra structural arterial stiffening and which may also related to the atherosclerotic vascular damage.

\section{Augmentation index (ai) and central brachial pressure (bp)}

The AI is used for measurements of the stiffness of the systemic

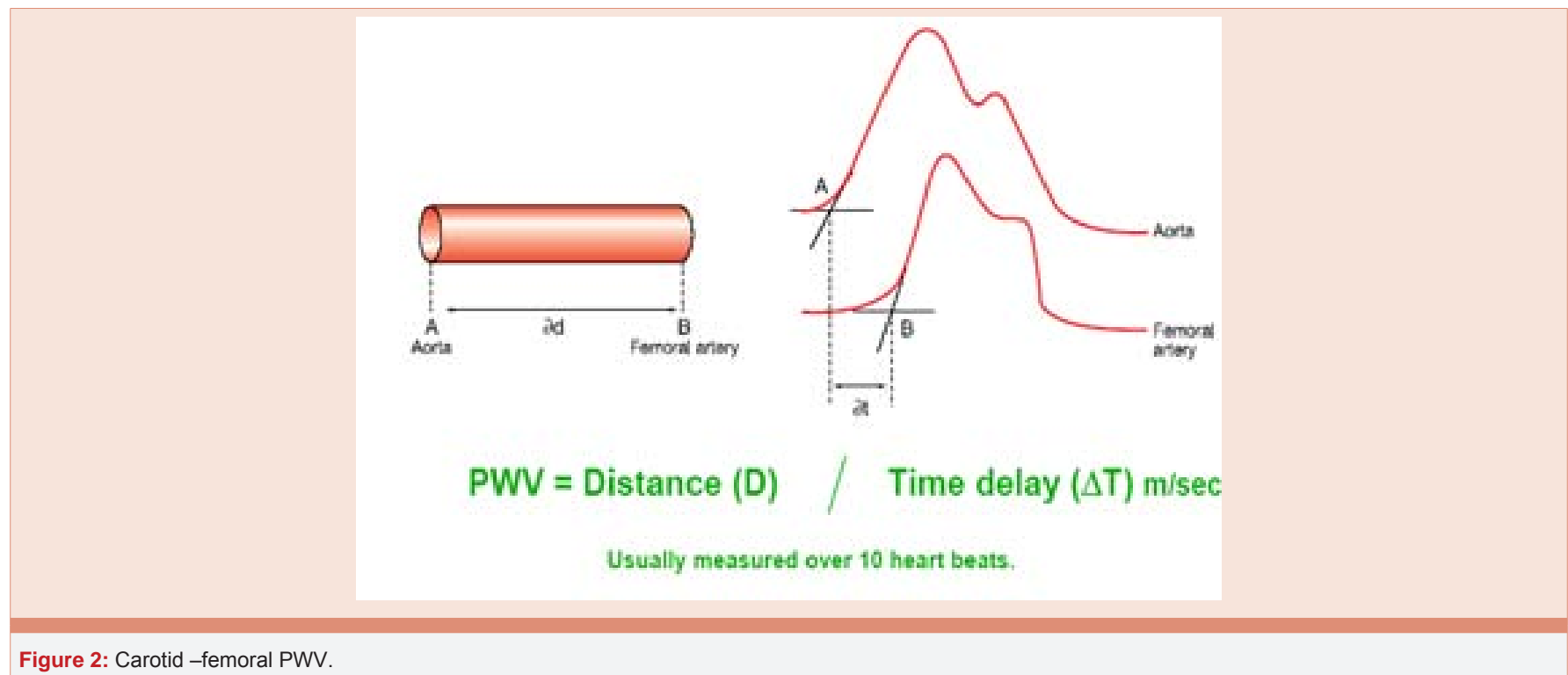

Figure 2: Carotid -femoral PWV. 


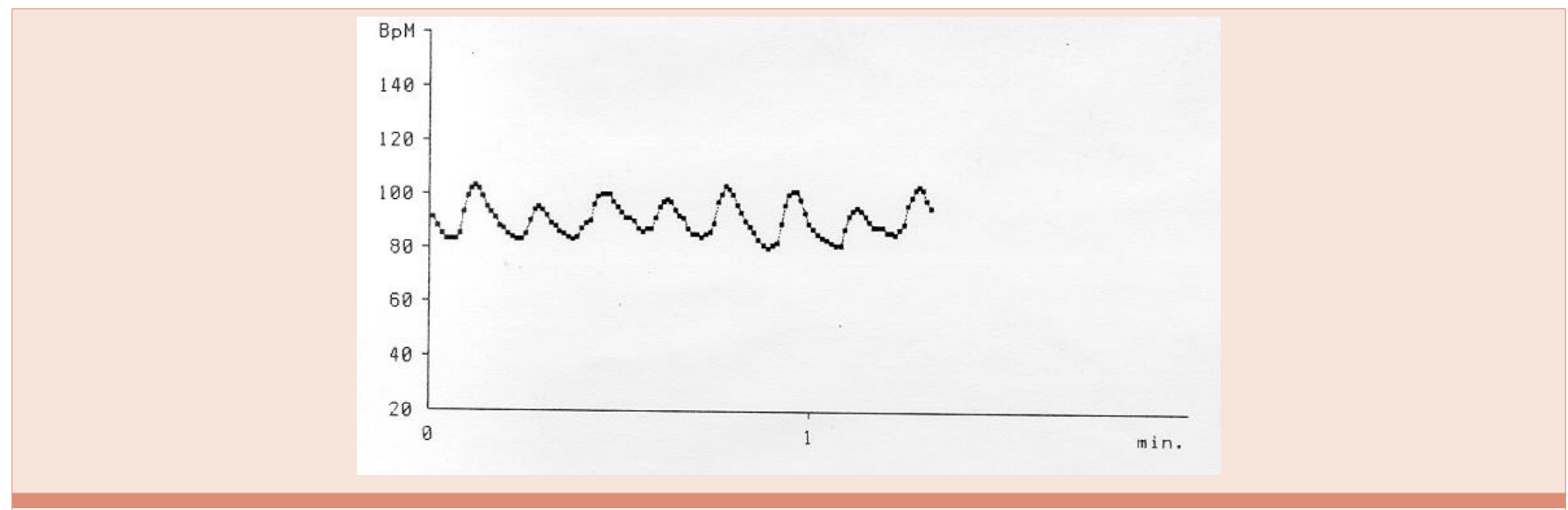

Figure 3: PWV in healthy volunteer he heart is the pump of the circulation.

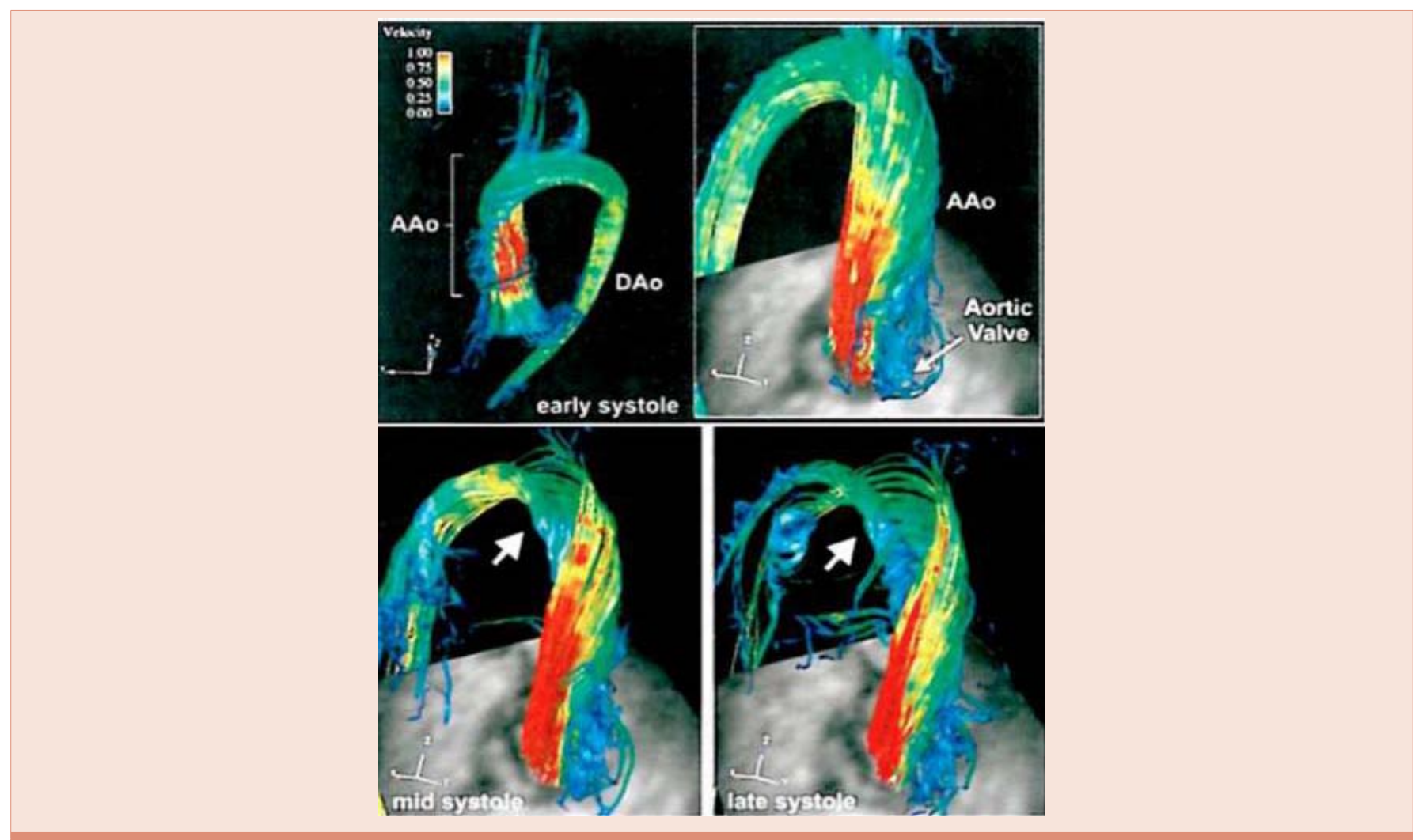

Figure 4: Flow pattern in aorta, so it Right -handed helical flow in the ascending aorta and arch during middle and late systole. Courtesy by Markl M., Draney MT, Miller DC et al. J Thorac. Cardiovascl Surg. 2005;130: 456-463.

arterial tree. In the arterial walls early changes are associated with an increase in arterial resistance. A reflected pulse wave (from the periphery to the heart) occurs at the sites of an abrupt increase in arterial resistance, such as arterial branching. The interaction or ratio between the incident pulse wave (from the heart to the periphery) and the reflected pulse wave (from the periphery to the central region) is assessed by pulse wave analysis and expressed as the AI $[22,40]$. Enhanced central arterial pressure waveform (PWV) and peripheral pulse reflectance are important determinants of the AI.
There is statistical relationship between increased central arterial stiffness and/or peripheral reflectance with an increase in central blood pressure and ages in population, decreases of the heart rate is associated with increase of AI and normally women are with higher AI that men [52]. In the clinical setting there are two non-invasive methods available to measure the AI. The first involves measurement of the AI at the carotid artery (carotid AI) and the other involves its measurement at the radial artery (radial AI); central BP can be non-invasively estimated from these recordings. Central (aortic 
and carotid) pressures are pathophysiologically more related than peripheral pressures in the outcomes of CVD [53]. Conventionally, increased stiffness in the arterial wall is related to increase the speed of the pulse wave and to shift the reflection point and increase the AI too [54]. The macro vascular dysfunction is related to the micro vascular dysfunction as part of the endothelial dysfunction. Higher AI is related with more target organ injures. In different studies with healthy volunteers and hypertensive patients were found that the carotidfemoral PWV is a predictor of future cardiovascular events $[55,56]$ and the brachial- ankle PWV could be a good marker of prognosis in subjects with acute coronary syndromes, end-stage renal disease or heart failure [57,58]. Aging, BP and dyslipidemia, diabetes causes vascular damages and increase the stiffening of the arteries $[59,60]$. Arterial stiffness parameter are closely associated with conventional risk factors and has been influenced by mean BP [61,62], which is demonstrated that structural arterial stiffening may also be related to atherosclerotic vascular damage. Last year several other methods (ie, ultrasound examination, computed tomography and/ or MRI) have been proposed for assessing the severity of atherosclerosis [64]. The PWV is correlated significantly and positively with atherosclerotic injures of the arterial wall and is one of the major markers for assessment the risk of CVD $[65,66]$ and together with AI are more related with to the increased risk of CVD associated with elastic artery stiffness. The stiffness index- $\beta$, is a marker of arterial stiffness with minimal influence of BP and were established as a marker of regional arterial stiffness [67]. Beta can be measured at the brachial, femoral or carotid artery, or at the aorta, after adjustment for BP in the different regions. Correlating with PWV is very important to know that beta is not useful for segmental arterial stiffness, because BP differs between the proximal and distal sites. Some studies have shown that each noninvasively increased $\mathrm{AI}$ and/ or central $\mathrm{BP}$ of 10 increase the adjusted overall risk in subjects with end-stage renal disease or coronary heart disease, American- Indians and hypertensive subjects [68,69]. Age, sex and heart rate are important determinants for the AI [70,71] and has been shown in the CAFÉ study that hemodynamics data reduction of central $\mathrm{BP}$ is related to improvement of the prognosis. [59]. So, the AI and central BP may be promising as markers of the effect of interventions for risk factors of CVD on outcomes.

FMD is very useful for the progression of structural artery disease, and the PWV is reported as a predictor of new onset of hypertension $[72,73]$. All noninvasive vascular functional tests have the potential to predict progression of vascular damage and target organ damage.

\section{Carotid intima media thickness}

The thickening of the intima media is used in testing of extent of carotid atherosclerotic diseases and to analyzing the progression of the process. Usually this progression of IMT is $1-5$ microns per year. The increase of IMT above of the normal value is associated with higher risk of cardiovascular events. Contributing factors for IMT increase are hypertension, diabetes, smoking, obesity, dyslipidemia, ages and hereditary lifestyles the main objective of the method is detection of carotid atheroma in the earliest phases in patients with risk factors, but also monitoring of the progression of IMT after aggressively treatment. Asymptomatic patients with pathological IMT have the higher risk for stroke and myocardial infarction. The screening and detection of subclinical atherosclerosis allow us to achieve specialized care and reduce the frequency of occurrence of cardiovascular events $[74,75]$. Arterial stiffness is an independent marker to predict of cardiovascular events.

\section{Flow mediated vasodilatation ( $\mathrm{fmd}$ )}

FMD is an additional noninvasive technique using in the indirect assessment of the degree of endothelial dysfunction, applied at the brachial artery and applicable to patients with risk factors and very well reproducible as test. The analysis of the pathological results is correlated with the progression of arterial atherosclerosis. This method is reflected by the degree of oxidative stress and severity of risk factors or poor control of the disease $[31,40,74,76]$. The measurement technique is based on initial ultrasound detection (with a $7.5 \mathrm{MHz}$ linear transducer in 2D mode) of the brachial artery followed by radio frequency measurement of the mean diameter and spectral analysis of the baseline blood flow (in $\mathrm{cm} / \mathrm{second}$ ). Once these baseline measurements have been made, an inflatable cuff (with a defined length of $10 \mathrm{~cm}$ ) is placed around the proximal third of the arm and a $300 \mathrm{mmHg}$ occlusive pressure is applied for 5 minutes. The first step is to then measure the baseline brachial artery diameter and flow. The next step is to measure the brachial artery diameter and flow after reactive hyperemia. In case of pathology, a physiological 15-minute arterial recovery period is required before continuing to the second part of the procedure (only in patients presenting endothelial dysfunction diagnosed by radio frequency (FMD)).

The second part of the procedure is the reversibility test, which is used to assess endothelial function (FMD). The reversibility test with an FMV-NO donor is expressed as the percentage recovery of the arterial diameter after reactive hyperemia compared to the same artery's baseline diameter of the endothelial dysfunction [3,40,77-79]. FMD, the PWV and the AI reflect in different faces of the atherosclerotic process, underlying functional vascular damage. Witte et al reported from their meta-analysis that FMD is related to the principal cardiovascular risk factors only in low-risk populations [80].

McEniery et al., proposed that the AI might be a more sensitive marker of arterial stiffening and cardiovascular risk in younger individuals, whereas the aortic PWV is likely to be a better measure in older individuals [81].

New technical noninvasive tests last years were proposed for the detection of subclinical atherosclerosis with very encouraging results: EPIV and MRI velocimetry [82-84].

\section{Echo particle image velocimetry (EPIV)}

EPIV is a new technique in the detection of subclinical atherosclerosis, used in measurements of shear wall stress through vector analysis of blood flow velocities of the wall at the carotid bifurcation. It is a ultrasound method, which need a technical high-temporal resolution of $0.7 \mathrm{~ms}$ and a spatial resolution of $0.4 \mathrm{~mm}$, combining EPIV contrast with a conventional 2D echo method. This technique is a synthesis of two technologies: particle image velocimetry, using the vectors, detecting the blood flow and ultrasound contrast agent used is a product of contrast micro- 
bubbles, which allows a better study of velocities and stresses (Figure 4). The parameters analyzing via EPIV are the vascular speed profile, velocity vector, speed, maps, and hemodynamic blood flow to the wall shear stress (calculating the average shear stress in the arterial wall in dyne $/ \mathrm{cm}^{2}$ ). The standards used for the wall stress are $-20+5$ dyne/ $\mathrm{cm}^{2}$ and wall pathological stress $>200$ dyne $/ \mathrm{cm}^{2}$ [85-87]

The challenge with this type of imaging is the great of quantity of information regarding flow velocity and direction changes rapidly, especially around bifurcations and valves where rapid changes are found. For example, the vortex in the carotid bifurcation presented in Figure 4 appears after peak systole and disappears within 100-200 $\mathrm{ms}$ downstream as the velocity is around $0.4-0.6 \mathrm{~m} \mathrm{~s}^{-1}$. It is therefore often necessary to inspect slow moving cine-loops or to study still frames as shown in this article to perceive the wealth of information.

\section{Magnetic resonance imaging (mri) velocimetry}

MRI velocimetry is a very promising technique for the detection of subclinical atherosclerosis. This technique studies the velocity profile, shear rate, and shear flow stress of the arterial wall. It is a noninvasive technique, more sensitive and reliable than the EPIV (with the border error between the two techniques of $10 \%$ for MRI). MRI velocimetry has both advantages and disadvantages, due to its high temporal resolution; it is now considered a promising approach for the noninvasive assessment of changes in carotid wall thickness, a marker for subclinical atherosclerosis in young patients with high risk and abdominal adiposity [88-90].

The MRI velocimetry appears to have a wide-range of applications. These sequences provide accurate reproducible haemodynamic information when the acquisition settings and section planes are chosen carefully. They appear to be underused in common practice, partly because of a lack of large series comparing their use with that of conventional methods (invasive or non-invasive)

Traditional risk factors predict future cardiovascular events and are major determinants in the assessment of cardiovascular risk (Framingham score and Euro SCORE) and the choice of primary prevention strategy. Nowadays early diagnosis of cardiovascular disease is a challenge, and especially its management along with a decrease in mortality, the cardiovascular disease still remains a major cause of morbidity and mortality of coronary in 50\% of men and $64 \%$ of women. In future years, there will be a further increase in the prevalence of cardiovascular disease secondary to the aging population and the rise of the percent of prevalence in obesity and diabetes. Currently the only available strategy to reduce mortality in this population is primary prevention in face of atherosclerosis screening. This approach in primary prevention is more effective when it is directed toward the early detection of subclinical atherosclerosis using new noninvasive imaging techniques that add value, not only in the screening of asymptomatic patients with risk factors, but also in the stratification of cardiovascular risk These new techniques allow us to have an overall picture of the state of physiological or pathological arterial remodeling and the degree of endothelial dysfunction. The results obtained with these techniques in the future will be associated with specific biomarkers to refine the diagnosis of subclinical atherosclerosis.

\section{Key Facts}

Assessment of the degree of subclinical atherosclerosis and particularly on arterial remodeling and endothelial function is available with different technics.

The use of duplex sonography for screening and early detection of subclinical atherosclerosis is reliable and reproducible and allows us to assess the sensitivity of the patients with risk factors for atherosclerosis and reducing the occurrence of cardiovascular events in the long term.

The new ultrasound parameters for assaying of vascular function are basically for the evaluation of the cardiovascular risk in patients with asymptomatic and symptomatic cardiovascular diseases.

The ultrasounds techniques allow to analyzing changes according to age and measure the pathological value as the thickness of the intima media, the speed of the pulse wave, and the degree of endothelial dysfunction. The screening is legitimate in asymptomatic patients with cardiovascular risk factors (hypertension, diabetes, obesity, dyslipidemia, and tobacco smoking).

\section{Summary Points}

Multiple risk factors are of major importance for the prevention of atherosclerosis and coronary artery diseases (CAD). However, it is clear that dyslipidemia plays a central role. The network of evidence is strongest for elevated levels of total cholesterol (TC) and low-density lipoprotein cholesterol (LDL-C). An inverse association has also been demonstrated between levels of high-density lipoprotein cholesterol (HDL-C) and its principal Apo lipoprotein, A-1, and risk of CAD.

Early diagnostic on the atherosclerosis in the human body will provide and early therapeutic lifestyle changes and effective therapeutic intervention has advanced the concept of primary prevention of coronary artery diseases. The purpose of this article is to review these contributions on all new noninvasive imaging techniques, with an emphasis on early changes on arteries and heart in patients with dyslipidemia.

\section{References}

1. Mihailidou AS, Ritchie R, Ashton AW (2013) Sex Differences in Sudden Cardiac Death, Ischemic Heart Disease, Dr. David Gaze (Ed.), InTech 61-75.

2. Castellon $X(2006)$ Methods of assessing endothelial function, vasodilation of interest by the intermediate flow. Cardiology Practice 8: 10-12.

3. Castellon X, Bogdanova V (2009) QIMT by radio frequency (examination of tracking), method of evaluation thickness of the intima media among patients having cardio vascular risk factors. Clinical implication: about 150 cases. Proceedings of the 4th International Conference on Oxidative/Nitrosative Stress and Disease; New York, NY, USA.

4. Charniot JC, Cosson C, Castellon X (2009) Comparison of oxidative stress status in patients with cardiogenic shock due to severe left ventricular dysfunction. Rejuv Res12: S17-S59.

5. Puddu P, Cravero E, Puddu M, Muscari A (2005) Genes and atherosclerosis: at the origin of the predisposition. Int J Clin Pract 59: 462-472.

6. Castellon X, Bogdanova V (2011) Study of arterial rigidity QAS. Arch Cardiovasc Dis 3: 85-86.

7. Richard JL, Ducimetiere P, Cambien F (1979) Tobacco, mortality and 
morbidity of atherosclerotic cardiovascular diseases - a prospective study in Paris. Bull Schweiz Akad Med Wiss 35: 51-69.

8. Charniot JC, Albertini JP, Cosson C (2010) 098 Mid-term follow up of consecutive patients with cardiogenic shock without acute coronary syndrome. Archives of Cardiovascular Diseases Supplements 2: 33

9. Castellon X, Bogdanova V (2011) Protocol of screening of sub clinical atherosclerosis by radio frequency (RF) coupled with 2D echo. Proceedings of the 5th: Conference of SENS; 2011 Aug 31-Sep 4; Cambridge, UK

10. Charniot JC, Vignat N, Albertini JP, Bogdanova V, Zerhouni K, et al. (2008) Oxidative stress in patients with acute heart failure. Rejuvenation Res 11: 393-398.

11. Bampi AB, Rochitte CE, Favarato D, Lemos PA, da Luz PL (2009) Comparison of non-invasive methods for the detection of coronary atherosclerosis. Clinics (Sao Paulo) 64: 675-682

12. Alexander RW (1995) Theodore Cooper Memorial Lecture. Hypertension and the pathogenesis of atherosclerosis. Oxidative stress and the mediation of arterial inflammatory response: a new perspective. Hypertension 25: 155161.

13. Berenson GS, McMahan CA, Voors AW (1980) Cardiovascular Risk Factors In Children: The Early Natural History Of Atherosclerosis And Essential Hypertension. New York, NY: Oxford University Press.

14. Everett Schultz Jr. (1998) Tobacco smoke and atherosclerosis progression. JAMA 280: 32-33.

15. Haapanen A, Koskenvuo M, Kesäniemi YA, Heikkilä K (1989) Carotid arteriosclerosis in identical twins discordant for cigarette smoking. Circulation 80: 10-16.

16. Conlan MG , Folsom AR, Finch A, Davis CE , Sorlie P, et al. (1993) Associations of factor VIII and von Willebrand factor with age, race, sex, and risk factors for atherosclerosis. The Atherosclerosis Risk in Communities (ARIC) Study. Thromb Haemost 70: 380-385.

17. Samelson EJ1, Booth SL, Fox CS, Tucker KL, Wang TJ, et al. (2012) Calcium intake is not associated with increased coronary artery calcification: the Framingham Study. Am J Clin Nutr 96: 1274-1280.

18. Zheng HR, Wang J (2008) A new velocimetry algorithm for optimizing ultrasonic PIV imaging method. Proceedings of the International Special Topic Conference on Information. Technology and Applications in Biomedicine 164166

19. Berenson GS, Srinivasan SR, Bao W, Newman WP, Tracy RE, et al. (1998) Association between multiple cardiovascular risk factors and atherosclerosis in children and young adults. The Bogalusa Heart Study. N Engl J Med 338 : 1650-1656.

20. Grimbone MA Jr. (1995) Vascular endothelium: an integrator of pathophysiologic stimuli in atherosclerosis. Am J Cardiol 75: 67B-70B.

21. Cyrus T1, Witztum JL, Rader DJ, Tangirala R, Fazio S, et al. (1999) Disruption of the 12/15-lipoxygenase gene diminishes atherosclerosis in apo E-deficient mice. J Clin Invest 103: 1597-1604.

22. O'Rourke MF, Gallagher DE (1996) Pulse wave analysis. J Hypertens 14 S147-S157.

23. Charniot JC, Cosson X, Castellon V (2009) Comparison of oxidative stress status in patients cardiogenic shock due to severe left ventricular dysfunction. Proceedings of the 4 th Conference of SENS. Cambridge, UK.

24. Abbott RD, Wilson PW, Kannel WB, Castelli WP (1988) High density lipoprotein cholesterol, total cholesterol screening, and myocardial infarction. The Framingham Study. Arteriosclerosis 8: 207-211.

25. Huck CJ, Bronas UG, Williamson EB, Draheim CC, Duprez DA, et al (2007) Noninvasive measurements of arterial stiffness: repeatability and interrelationships with endothelial function and arterial morphology measures. Vasc Health Risk Manag 3: 343-349.

26. Ruiz-Ortega M, Lorenzo O, Rupérez M (2001) Fourth International Seminar on Cardiovascular Biology and Medicine: Part II. Role of the renin-angiotensin system in vascular diseases. Expanding the field. Hypertension 38: 1382 1387.

27. Touyz RM (2005) Intracellular mechanisms involved in vascular remodelling of resistance arteries in hypertension: role of angiotensin II. Exp Physiol 90: $449-455$.

28. Revkin JH, Shear CL, Pouleur HG, Ryder SW, Orloff DG (2007) Biomarkers in the prevention and treatment of atherosclerosis: need, validation, and future. Pharmacol Rev 59: 40-53.

29. Ridker PM, Stampfer MJ, Rifai N (2001) Novel risk factors for systemic atherosclerosis: a comparison of C-reactive protein, fibrinogen, homocysteine, lipoprotein(a), and standard cholesterol screening as predictors of peripheral arterial disease. JAMA 285: 2481-2485.

30. Wissler RW, Strong JP (1998) Risk factors and progression of atherosclerosis in youth. PDAY Research Group. Pathological Determinants of Atherosclerosis in Youth. Am JPathol 153: 1023-1033.

31. Schwartz CJ, Valente AJ, Sprague EA, Kelley JL, Cayatte AJ, et al. (1992) Atherosclerosis. Potential targets for stabilization and regression. Circulation 86: III117-123.

32. Charniot JC, Vignat N, Albertini JP, Bogdanova V, Zerhouni K, et al. (2008) Oxidative stress implication in acute heart failure. Rejuvenation Res 11: 393398.

33. Castellon X, Bogdanova V, Charniot JC (2009) QIMT by radio frequency (examination of tracking). Method of evaluation thickness of the intima media among patients having cardio vascular risk factors. Proceedings of the 4 th Conference of SENS; Sep 3-7; Cambridge, UK.

34. Vanauker MD, Tacy TA, del Nido PJ, Cape EG (2001) Development of a noninvasive marker of wall shear stress effects in discrete subaortic stenosis. Cardiovascular Engineering 1: 137-146.

35. Li JJ, Fang CH (2004) C-reactive protein is not only an inflammatory marker but also a direct cause of cardiovascular diseases. Med Hypotheses 62: 499506.

36. Wang SS1, Schadt EE, Wang H, Wang X, Ingram-Drake L, et al. (2007) Identification of pathways for atherosclerosis in mice: integration of quantitative trait locus analysis and global gene expression data. Circ Res 101: e11-e30.

37. Shaaban AM, Duerinckx AJ (2000) Wall shear stress and early atherosclerosis a review. AJR Am J Roentgenol 174: 1657-1665.

38. Cohen JC, Zannis VI (2001) Genes affecting atherosclerosis. Curr Opin Lipidol 12: 93-95

39. Cunningham KS, Gotlieb Al (2005) The role of shear stress in the pathogenesis of atherosclerosis. Lab Invest 85: 9-23.

40. Reiner Z, Tedeschi-Reiner E (2001) New information on the pathophysiology of atherosclerosis. Lijec Vjesn 123: 26-31.

41. Gutierrez MA, Higa M, Pilon PE, Rebelo MDS, Lage SG (2013) Quantitative Assessment of Peripheral Arteries in Ultrasound Images. Medical Imaging in Clinical Practice 234-255.

42. Charniot JC, Cosson C, Chemouni F, Vignat N, Bogdanova V, et al. (2010) 087 Oxidative stress implications in cardiogenic shock with ischemic or idiopathic severe left ventricular dysfunction: role of etiologies of cardiomyopathies. Archives of Cardiovascular Diseases Supplements 2: 29.

43. Castellon X, Bogdanova V (2009) Analysis of endothelial function by radiofrequency coupled with two-dimensional echocardiography: the value of flow-mediated vasodilatation. A study of 25 cases. Proceedings of the 4 th Conference of SENS, Cambridge, UK.

44. Falk E (2006) Pathogenesis of atherosclerosis. J Am Coll Cardiol 47: C7C12.

45. Rutter MK, Meigs JB, Sullivan LM, D’Agostino RB, Sr, Wilson PW (2004) 
C-reactive protein, the metabolic syndrome, and prediction of cardiovascular events in the Framingham Offspring Study. Circulation 110: 380-385.

46. Kim T, Seo JH, Bang SS, Choi HW, Chang Y, et al. (2010) Optimisation de la vélocimétrie MR flux de contraste de phase basée sur des mesures et des contraintes de cisaillement. [Optimization of MR phase-contrast-based flow velocimetry and shear stress measurements]. Int J Cardiovasc Imaging 26 : 133-142.

47. Tsao CW, Preis SR, Peloso GM, Hwang SJ, Kathiresan S, et al. (2012) Relations of long-term and contemporary lipid levels and lipid genetic risk scores with coronary artery calcium in the framingham heart study. J Am Coll Cardiol 60: 2364-2371.

48. Toth PP (2008) Subclinical atherosclerosis: what it is, what it means and what we can do about it. Int J Clin Pract 62: 1246-1254.

49. Brindle P, Emberson J, Lampe F, Walker M, Whincup P, et al. (2003) Predictive accuracy of the Framingham coronary risk score in British men: prospective cohort study. BMJ 327: 1267.

50. Lusis AJ, Mar R, Pajukanta P (2004) Genetics of atherosclerosis. Annu Rev Genomics Hum Genet 5: 189-218.

51. Lakoski SG, Greenland P, Wong ND, Schreiner PJ, Herrington DM, et al (2007) Coronary artery calcium scores and risk for cardiovascular events in women classified as "low risk" based on Framingham risk score: the multiethnic study of atherosclerosis (MESA). Arch Intern Med 167: 2437-2442.

52. Abbott RD, Wilson PW, Kannel WB, Castelli WP (1998) High density lipoprotein cholesterol, total cholesterol screening and myocardial infarction. The Framingham Study. Atherosclerosis 8: 207-211.

53. Boutouyrie P, Tropeano Al, Asmar R, Gautier I, Benetos A, et al. (2002) Aortic stiffness is an independent predictor of primary coronary events in hypertensive patients. Hypertension 39: 10-15

54. Lagrand WK, Visser CA, Hermens WT, Niessen HW, Verheugt FW, et al (1999) C-reactive protein as a cardiovascular risk factor: more than an epiphenomenon? Circulation 100: 96-102.

55. Widlansky ME, Gokce N, Keaney JF, Vita JA (2003) Les implications cliniques de la dysfonction endothéliale. [The clinical implications of endothelial dysfunction]. J Am Coll Cardiol 42: 1149-1160.

56. Tsou JK, Liu J, Insana MF (2006) Modeling and phantom studies of ultrasonic wall shear rate measurements using coded pulse excitation. IEEE Trans Ultrason Ferroelectr Freq Control 53: 724-734

57. Sengupta PP, Pedrizzetti G, Kilner PJ, Kheradvar A, Ebbers T, et al. (2012) Emerging trends in CV flow visualization. JACC Cardiovasc Imaging 5: 305316

58. Adji A, O'Rourke MF, Namasivayam M (2011) Arterial stiffness, its assessment, prognostic value, and implications for treatment. Am J Hypertens 24: 5-17.

59. Laurent S, Cockcroft J, Van Bortel L, Boutouyrie P, Giannattasio C, et al (2006) European Network for Non-invasive Investigation of Large Arteries. Expert consensus document on arterial stiffness: methodological issues and clinical applications. Eur Heart J 27: 2588-2605.

60. Calermajer DS, Sorensen KE, Gooch VM, Spiegelhalter DJ, Miller OI, et al. (1992) Non-invasive detection of endothelial dysfunction in children and adults at risk of atherosclerosis. Lancet 340: 1111-1115.

61. Bouhanick B, Chamontin B (2007) Should pulse pressure and day/night variations in blood pressure be seen as independent risk factors requiring correction or simply as markers to be taken into account when evaluating overall vascular risk? Diabetes Metab 33: 321-330.

62. Meaume S, Benetos A, Henry OF, Rudnichi A, Safar ME (2001) Aortic pulse wave velocity predicts cardiovascular mortality in subjects $>70$ years of age. Arterioscler Thromb Vasc Biol 21: 2046-2050.

63. Cockcroft JR, Wilkinson IB (1979) Arterial stiffness and pulse contour analysis: an age old concept revisited. Clinical Science 103: 379-380.
64. Laurent S, Kingwell B, Bank A, Weber M, Struijker-Boudier H (2002) Clinical applications of arterial stiffness: therapeutics and pharmacology. Am J Hypertens 15: 453-458.

65. Kataoka Y, Shao M, Wolski K, Uno K, Puri R, et al. (2012) Multiple risk factor intervention and progression of coronary atherosclerosis in patients with type 2 diabetes mellitus. Eur J Prev Cardiol 20: 209-217.

66. Mackenzie IS, Wilkinson IB, Cockcroft JR (2002) Assessment of arterial stiffness in clinical practice. QJM 95: 67-74.

67. Schiffrin EL, Touyz RM (2004) From bedside to bench to bedside: role of renin-angiotensin-aldosterone system in remodeling of resistance arteries in hypertension. Am J Physiol Heart Circ Physiol 287: H435-H446.

68. Libby P, Ridker PM, Maseri A (2002) Clinical cardiology: new frontiers. Inflammation and atherosclerosis. Circulation 105: 1135-1143.

69. Berliner JA, Navab M, Fogelman AM, Frank JS, Demer LL, et al. (1995) Atherosclerosis: basic mechanisms. Oxidation, inflammation, and genetics. Circulation 91: 2488-2496.

70. Franklin SS, Gustin W 4th, Wong ND, Larson MG, Weber MA, et al. (1997) Hemodynamic patterns of age-related changes in blood pressure. The Framingham Heart Study. Circulation 96: 308-315.

71. Towfighi A, Markovic D, Ovbiagele B (2012) Utility of Framingham Coronary Heart Disease Risk Score for predicting cardiac risk after stroke. Stroke 43 2942-2947.

72. Touboul PJ, Hennerici MG, Meairs S, Adams H, Amarenco P, et al. (2007) Mannheim carotid intima-media thickness consensus (2004-2006). An update on behalf of the Advisory Board of the 3rd and 4th Watching the Risk Symposium, 13th and 15th European Stroke Conferences, Mannheim, Germany, 2004, and Brussels, Belgium, 2006. Cerebrovasc Dis 23: 75-80.

73. Nicholls SJ, Tuzcu EM, Crowe T, Sipahi I, Schoenhagen P, et al. (2006) Relationship between cardiovascular risk factors and atherosclerotic disease burden measured by intravascular ultrasound. J Am Coll Cardiol 47: $1967-$ 1975.

74. Lane HA, Smith JC, Davies JS (2006) Noninvasive assessment of preclinical atherosclerosis. Vasc Health Risk Manag. 219(1):30.

75. Nillson PM, Boutouyrie P, Laurent S (2009) Vascular aging: a tale of EVA and ADAM in cardiovascular risk assessment and prevention. Hypertension 54: 3-10.

76. Castellon X, Bogdanova V (2010) QIMT by radio frequency (examination of tracking). Method of evaluation thickness of the intimae media among patients having cardio vascular risk factors. Proceedings of Euro Prevent Conference; May 5-7; Prague, Czech Republic.

77. Castellon X, Bogdanova V (2012) Screening protocol of subclinical atherosclerosis with noninvasive imaging methods, by radio frequency (RF) echo 2D. Arch Cardiovasc Dis 4: 49.

78. Castellon X, Bogdanova V (2011) Screening protocol of sub clinical atherosclerosis by radio frequency (RF) coupled with $2 \mathrm{~d}$ echo. Proceedings of the Conference of Evolving Challenges in Promoting Cardiovascular Health, Barcelona, Spain.

79. Stocker R, Keaney JF Jr. (2004) Role of oxidative modifications in atherosclerosis. Physiol Rev 84: 1381-1478.

80. Tomiyama H, Yamashina A, Arai T, Hirose K, Koji Y, et al. (2010) Influences of age and gender on results of noninvasive brachial-ankle pulse wave velocity measurement-a survey of 12517 subjects. Atherosclerosis 166: 303-330.

81. Intengan HD, Schiffrin EL (2001) Vascular remodeling in hypertension: roles of apoptosis, inflammation, and fibrosis. Hypertension 38: 581-587.

82. Sung KC, Kang SM, Cho EJ, Park JB, Wild SH, et al. (2012) Ferritin is independently associated with the presence of coronary artery calcium in 12,033 men. Arterioscler Thromb Vasc Biol 32: 2525-2530.

83. Blankenberg S, Barbaux S, Tiret L (2003) Adhesion molecules and atherosclerosis. Atherosclerosis 170: 191-203. 
84. Howard G, Wagenknecht LE, Burke GL, Diez-Roux A, Evans GW, et al. (1998) Cigarette smoking and progression of atherosclerosis: The Atherosclerosis Risk in Communities (ARIC) Study JAMA 279: 119-124.

85. Harrison D, Griendling KK, Landmesser U, Hornig B, Drexler H (2003) Role of oxidative stress in atherosclerosis. Am $\mathrm{J}$ Cardiol 91: 7A-11A.

86. Kullo IJ, Gau FT, Tajik AJ (2000) Novel risk factors for atherosclerosis. Mayo Clin Proc 75: 369-380.

87. Singh U, Jialal I (2006) Oxidative stress and atherosclerosis. Pathophysiology 13: $129-142$.

88. Simon A, Gariepy J, Levenson J (1997) Ultrasonographic study of the arterial walls: application to the detection of preclinical atherosclerosis. Arch Mal Coeur Vaisseaux 90: 7-10.

89. Duprez DA (2006) Role of the rennin-angiogenesis-aldosterone system in vascular remodelling and inflammation: a clinical review. J Hypertens 24: 983-991.
90. Marmot MG, Rose G, Shipley MJ, Thomas BJ (1981) Alcohol and mortality: a U-shaped curve. Lancet 1: 580-583.

91. Mannucci PM (1998) von Willebrand factor: a marker of endothelial damage? Arterioscler Thromb Vasc Biol 18: 1359-1362.

92. Lange RA (2013) Can you predict what happens when EuroSCORE weds biomarker? J Am Coll Cardiol 61: 682-684.

93. Robertson MB, Köhler U, Hoskins PR, Marshall I (2001) Quantitative analysis of PC MRI velocity maps: pulsatile flow in cylindrical vessel. Magn Reson Imaging 19: 685-695.

94. Shaw LJ, Raggi P, Callister TQ, Berman DS (2006) Prognostic value of coronary artery calcium screening, in asymptomatic smokers and nonsmokers. Eur Heart J 27: 968-975.

Copyright: (c) 2016 Hristova K. This is an open-access article distributed under the terms of the Creative Commons Attribution License, which permits unrestricted use, distribution, and reproduction in any medium, provided the original author and source are credited. 